\title{
Generalized annular pustular psoriasis (Milian Katchoura type) induced by systemic steroid
}

\author{
Madiha El Jazouly ${ }^{1}$, Maha ALJ ${ }^{1}$, Fatim zahra Chahboune ${ }^{1}$, Abderrahmane AL BOUZIDI ${ }^{2}$, \\ and Soumiya Chiheb ${ }^{1}$ \\ ${ }^{1}$ Cheikh Khalifa International University Hospital \\ ${ }^{2}$ Military hospital Mohammed V, Rabat, Morocco
}

January 20, 2021

\begin{abstract}
Pustular psoriasis is a rare, acute form of psoriasis. It can be triggered by pregnacy, infection, hypocalcemia, and medications. We describe a case of annular pustular psoriasis Milian Katchoura type, a rare variant of pustular psoriasis, that occurred secondary to reducing the dose of oral corticosteroids.
\end{abstract}

\section{Generalized annular pustular psoriasis (Milian Katchoura type) induced by systemic steroid}

Madiha El Jazouly, Maha Alj, Fatimzahra Chahboun, Abderrahman Bouzidi, Soumiya Chiheb

1 Department of Dermatology, Cheikh Khalifa internationnal Hospital

2 Department of Pathology, Cheikh Khalifa internationnal Hospital

University Mohamed IV of Health Sciences Casablanca Morocco

Corresponding author : El Jazouly Madiha

Keywords : Subcorneal pustular dermatosis, Annular gereralized pustular psoriasis, Sneddon Wilkhinson desease

Abstract :

Pustular psoriasis is a rare, acute form of psoriasis. It can be triggered by pregnacy, infection, hypocalcemia, and medications. We describe a case of annular pustular psoriasis Milian Katchoura type, a rare variant of pustular psoriasis, that occurred secondary to reducing the dose of oral corticosteroids.

Clinical image:

A 56 years old female patient with a 5-year history of plaque psoriasis treated with topical corticostreoids and phototherapy was admitted to the Department of Dermatology for a generalized rush which appeared after she reduced the dose of an oral treatment based on $60 \mathrm{mg}$ of prednisone. Dermatological examination revealed well-demarcated, large, annular erythematous and squamous lesions (Figure1), with peripheral micro- pustules. The polycyclic plaques was paler centrally surrounded by raised ring, confluent numerous pustules with trailing scale (Figure 2). The patient had no systemic symptoms or fever. Laboratory tests revealed a biological inflammatory syndrome with a normal calcemia and a sterile pustule at the bacterial sampling. Several diagnostics have been suggested: acute generalized exanthematous pustulosis, annular pustular psoriasis and subcorneal pustular dermatosis. 
Histopathological examination revealed a subcorneal spongiform pustule, acanthosis with hyperkeratosis and parakeratosis. In the dermis, there was a mononuclear cells and neutrophils infiltrate (Figure3).

The diagnosis of generelized annular pustular psoriasis (Millian Katchoura type) was retained because of the personal history of psoriasis, the evocative clinical aspect, and the histological findings. The patient was treated with acitretin $10 \mathrm{mg} / \mathrm{kg} /$ day with a total regression of lesions.

Annular pustular psoriasis, described by Milian and Katchoura in 1933, is a rare form of pustular psoriasis. The pustular psoriasis may be induced or worsen by a number of factors primarily drugs such as lithium, beta blockers, terbinafine, biotherapy, hydroxychloroquine and a rapid taper of oral corticosteroids $(1,2)$. In order to avoid serious complications such as the occurrence of pustular forms of psoriasis, systemic corticosteroids should be prescribed with great caution to the patients.

Legends:

Figure1: Annular erythematous and squamous lesions

Figure2: Poly-cyclic pustular and papular lesions with trailing scale

Figure 3: subcorneal spongiform pustule, a mononuclear and neutrophils cell infiltrate the dermis (Hematoxylin and eosin $\mathrm{x} 200)$

Reference :

Westphal DC, Schettini APM, Souza PP, Castiel J, Chirano CA, Santos M. Generalized pustular psoriasis induced by systemic steroid dose reduction. An Bras Dermatol. 2016;91(5):664-6.

Du-Thanh A, Girard C, Pageaux GP, Guillot B, Dereure O. Sorafenib-induced annular pustular psoriasis (Milian-Katchoura type). EJD, vol. 23, n* 6, November-December 2013

CONFLICT OF INTEREST

No conflicting interest.

\section{AUTHOR CONTRIBUTION}

MEJ: is a corresponding author of the manuscript AND wrote the manuscript. AM and FZC: involved in photograph taking. AB: contributed to histological examination.SC: supervised the manuscript.

\section{Hosted file}

figure_1.pdf available at https://authorea.com/users/390810/articles/505037-generalizedannular-pustular-psoriasis-milian-katchoura-type-induced-by-systemic-steroid 


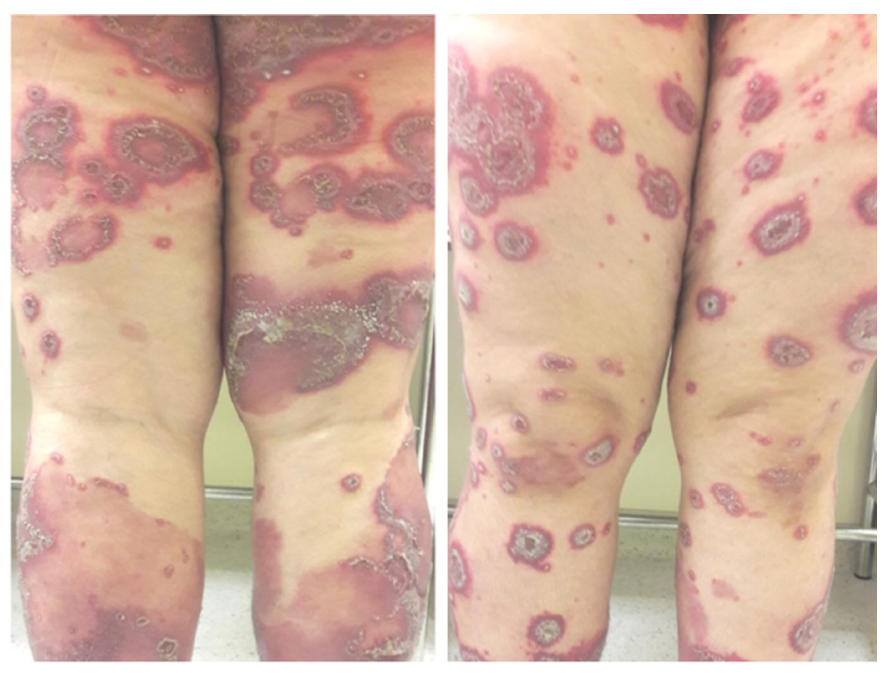




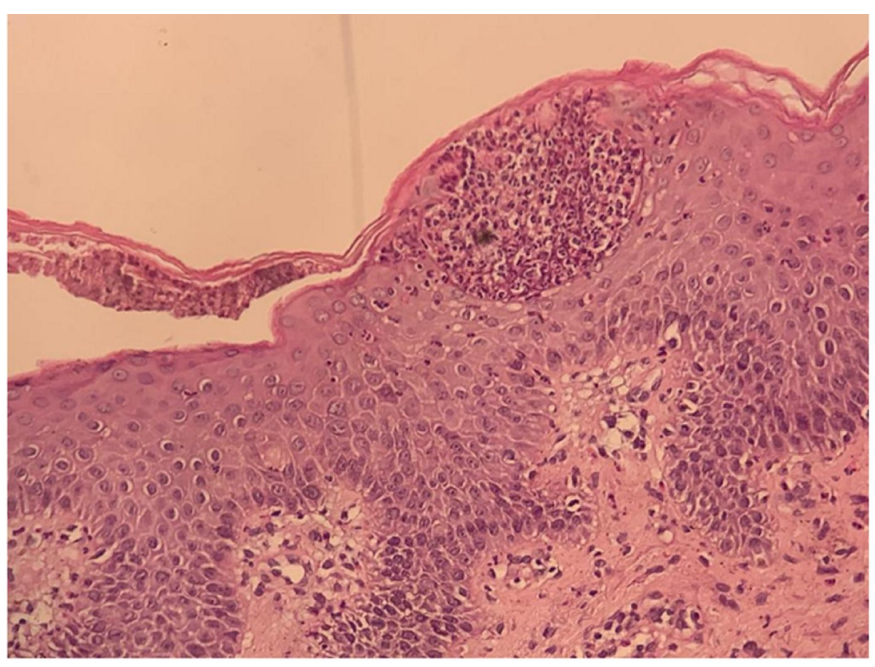

